Shubhang K. Bhatt, BS, Andrew S. Tseng, MD, Christine Firth, MD, Marlene Girardo, MS, Daniel Sykora, BA, Mina Abdelmalek, MD, F. David Fortuin, MD, Paul Wennberg, MD, David Liedl, RN and Fadi E. Shamoun*, MD

\title{
Abnormal vascular physiology in the lower extremities as a risk factor for ischemic stroke and mortality
}

https://doi.org/10.1515/jom-2020-0290

Received November 9, 2020; accepted December 22, 2020; published online March 11, 2021

\begin{abstract}
Context: Peripheral artery disease (PAD) is highly prevalent in the general population, affecting up to $25 \%$ of patients 55 years of age or older. There is a known association with acute ischemic stroke, but limited large cohort studies exist pertaining to the relationship between PAD severity and incident ischemic stroke.

Objectives: To evaluate the risk of incident ischemic stroke and mortality along the spectrum of low and elevated ankle brachial index (ABI) measurement.

Methods: We performed a retrospective extraction of $\mathrm{ABI}$ data of all adult patients who underwent lower extremity physiology study for any indication from January 1, 1996 to June 30, 2018 in the Mayo Clinic health system. PAD was categorized into severe, moderate, mild, and borderline based on ABI measurements and poorly compressible arteries (PCA). These were compared with normal ABI measurements. Associations of PAD/PCA with new ischemic
\end{abstract}

*Corresponding author: Fadi E. Shamoun, MD, Department of Cardiovascular Diseases at the Mayo Clinic, Phoenix, AZ, USA; and Mayo Clinic, 13400 E Shea Blvd, Scottsdale, AZ 85259-5452, USA, E-mail: shamoun.fadi@mayo.edu

Shubhang K. Bhatt, BS and Daniel Sykora, BA, Mayo Clinic Alix School of Medicine, Scottsdale, AZ, USA

Andrew S. Tseng, MD, Paul Wennberg, MD and David Liedl, RN, Department of Cardiovascular Diseases at the Mayo Clinic, Rochester, MN, USA

Christine Firth, MD, Mina Abdelmalek, MD and F. David Fortuin, MD, Department of Cardiovascular Diseases at the Mayo Clinic, Phoenix, AZ, USA

Marlene Girardo, MS, Department of Biomedical Statistics and Informatics, Division of Health Sciences Research at the Mayo Clinic, Scottsdale, AZ, USA stroke events and all cause mortality were analyzed. Hazard ratios (HR) were calculated using multivariable Cox proportional regression with $95 \%$ confidence intervals.

Results: A total of 39,834 unique patients were included with a median follow up duration of 4.59 years. All abnormal ABI groups, except borderline PAD, were associated with increased risk of incident ischemic stroke after multivariate regression compared to normal ABI. A severity-dependent association was observed between PAD and ischemic stroke with moderate (HR, 1.22 [95\% CI, 1.10-1.35]) and severe (HR, 1.19 [95\% CI, 1.02-1.40]) categories conferring similar risk in comparison to normal ABI. Patients with PCA carried the greatest ischemic stroke risk (HR, 1.30 [95\% CI, 1.15-1.46]). Similarly, abnormal ABI groups were associated with a significant risk for all cause mortality in a severity-dependent manner, with severe PAD conferring the greatest risk (HR, 3.07 [95\% CI, 2.88-3.27]). Conclusions: This study adds to the growing body of evidence that both PAD and PCA are independent risk factors for incident ischemic stroke and all cause mortality. The association of PAD severity and PCA with risk of ischemic stroke may help clinicians with risk stratification and determining treatment intensity.

Keywords: ankle brachial index; ischemic stroke; mortality; peripheral artery disease; poorly compressible arteries.

Peripheral artery disease (PAD) is pervasive among the general population, occurring in $15-25 \%$ of adults age 55 years and older [1]. Despite its high prevalence, PAD remains underdiagnosed, limiting the opportunity for early and more effective intervention and risk modification for associated cardiovascular (CV) comorbidities [2]. The ankle brachial index (ABI) is a reliable and noninvasive method to assess lower extremity physiology that can be readily performed in most clinical settings [3]. It can detect lower extremity arterial narrowing with specificity of 83.3-99.0\% and sensitivity of $15-79 \%$ for ABI cutoff of $\leq 0.9$ [3]. An 
ABI $\leq 0.9$ highly predicts recurrent ischemic stroke outcomes in patients with prior ischemic stroke $[4,5]$.

PAD is prevalent among $44.9 \%$ of patients with an acute ischemic stroke [6]. Prior studies have shown an association between low ABI and incident stroke $[1,7]$ as well as other adverse CV events, arterial ischemia, and all cause mortality [3, 8]. A metaanalysis by Hong et al. [3] revealed an increased risk of vascular events or vascular deaths as well as recurrent stroke in patients with low ABI. Although the existing literature shows an association between PAD severity and all cause mortality [9, 10], there are limited studies assessing the association between PAD severity and ischemic stroke in a large population cohort $[7,11]$.

Furthermore, conflicting evidence exists regarding the relationship between poorly compressible arteries (PCA) and stroke, which warrants further examination [10-13]. Patients with PCA are defined by stiff, non-compressible lower extremity vessels as a consequence of medial arterial calcification [14]. The stiffness of the vessels can lead to either an inability to obtain an ABI because of nonmeasurable blood pressure or a falsely elevated $\mathrm{ABI}>1.4$, and is referred to as PCA [14]. In comparison to healthy patients, arteries in patients with PAD but not PCA are associated with low compliance $[15,16]$. Studies have shown that the PCA group is associated with increased all cause mortality $[10,12]$, but the association with cardiovascular mortality, coronary artery events, or ischemic stroke has yielded mixed results $[13,17,18]$. Our study aimed to investigate the predictive value of different abnormal ABI groupings for incident ischemic stroke and all cause mortality in a large patient cohort.

\section{Methods}

\section{Study design}

We performed a retrospective cohort study to assess the relationship between different abnormal ABI groupings and incident ischemic stroke. The inclusion criterion was any adult patient with or without PAD/PCA symptoms who underwent ABI measurement for a clinically indicated reason in the Mayo Clinic health system between January 1, 1996 and June 30, 2018; patients below the age of 18 years were excluded. ABI categories were defined according to prior literature [19] and 2016 AHA/ACC PAD management guidelines [8]: 0.00-0.39, severe PAD; 0.40-0.69, moderate PAD; 0.70-0.90, mild PAD; 0.91-0.99, borderline PAD; 1.00-1.40, normal ABI; and greater than 1.4, PCA. While the primary outcome of interest was incident ischemic stroke, all cause mortality was also assessed. The study complied with the standards of and was approved by the Mayo Clinic Institutional Review Board. Informed consent requirement was waived due to the noninvasive nature of the study.
The assessment of lower extremity vascular physiology was performed by following standardized protocols in the noninvasive vascular laboratory certified by the Intersocietal Commission for the Accreditation of Vascular Laboratories [20]. A handheld doppler was used to measure systolic blood pressure (SBP) in the brachial artery on both arms. Similarly, SBP was measured in both the dorsalis pedis and posterior tibial arteries. For each lower extremity, the higher of the lower extremity SBPs was divided by the higher of the SBPs from the upper extremities to calculate the ABI. A patient was said to have a $\mathrm{PAD}$ or PCA if either side of their body had an $\mathrm{ABI}<1.0$ or $\mathrm{ABI}>1.4$.

\section{Comorbidity and outcomes variables}

All patient and outcomes variables were obtained using the institutional electronic health record (EHR). These variables were defined by the International Classification of Diseases (ICD) 9th [21] and 10th [22] revisions diagnostic codes. The ICD 9 and 10 codes used in this study are provided as Supplemental Material. The linking of the institutional EHR with the national United States Mortality Database accounted for the validity of our mortality outcomes.

\section{Statistical analysis}

Descriptive analysis was performed where continuous variables are described via their means and standard deviations, while categorical variables are described by their frequencies and percentages. We compared continuous variables using the analysis of variance (ANOVA) F-test, while categorical variables were compared using the chi-square test of significance. A p-value $<0.05$ was considered statistically significant.

Univariate and multivariate Cox proportional regression analysis was completed to assess hazard ratios (HR) with $95 \%$ confidence intervals (CI) for the outcomes of interest. Based on associations established in prior studies [4, 23], the variables adjusted in the multivariate model included age, gender, atrial fibrillation, chronic kidney disease stage $\geq 3$, history of ischemic stroke before index ABI, diabetes mellitus, hypertension, and carotid artery disease. Kaplan-Meier curves were computed for incident stroke by ABI groups. All statistical calculations were performed using SAS version 9.4 software (SAS institute Inc., Cary, NC).

\section{Results}

\section{Cohort demographics}

A total of 39,834 patients underwent ABI studies at any Mayo Clinic facility during the study period and were included over a median follow up of 4.59 years (interquartile range, 7.18 years). The low ABI group included 17,091 patients (42.9\%), the elevated ABI group included 5,071 patients (12.7\%), and the normal ABI group included 17,672 patients (44.4\%). Compared with patients with normal ABI, patients with low and elevated ABI were more likely $(p<0.05)$ to be older men with hypertension, hyperlipidemia, diabetes mellitus, transient ischemic attack (TIA), prior 
ischemic stroke, atrial fibrillation (AF), coronary artery disease, carotid artery disease, and/or chronic kidney disease stage 3 or greater (CKD 3+). Patients with low ABI were more likely $(\mathrm{p}<0.05)$ to be smokers whereas elevated ABI had lower prevalence of smokers compared to those with normal ABI. Baseline demographics and clinical characteristics of the patients included in this study are summarized in Table 1.

\section{Ischemic stroke incidence by $A B I$ group}

Ischemic stroke occurred in $7.2 \%(2,872)$ of the cohort during the study period. Furthermore, $8.3 \%(1,412)$ patients with low $\mathrm{ABI}, 8.9 \%$ (453) with elevated $\mathrm{ABI}$, and $5.7 \%(1,007)$ with normal $A B I$ suffered from ischemic stroke. AF, CKD 3+, history of ischemic stroke, hypertension, and carotid artery disease were independently associated with ischemic stroke. Univariate Cox proportional hazard analysis showed a statistically significant association between ischemic stroke and all abnormal $\mathrm{ABI}$ groups. The greatest risk with ischemic stroke was found in patients with severe PAD (HR, 1.73 [95\% CI, 1.47-2.02]) and PCA (HR, 2.03 [95\% CI, 1.82-2.27]). History of prior ischemic stroke conferred the greatest risk (HR, 11.48 [95\% CI, 10.52-12.52]) for future ischemic stroke.

After adjusting for the aforementioned variables in the multivariate model, all abnormal ABI groups (except borderline PAD) were independent predictors for future ischemic stroke (Figure 1). Compared with patients with normal ABI, PCA was associated with the greatest ischemic stroke risk (HR, 1.30 [95\% CI, 1.15-1.46]). A severitydependent relationship between PAD and ischemic stroke was observed except when the PAD worsened from the moderate (HR, 1.22 [95\% CI, 1.10-1.35]) to severe (HR, 1.19 [95\% CI, 1.02-1.40]) category. The risk for ischemic stroke was highest in patients with a history of prior ischemic stroke (HR, 8.71 [95\% CI, 7.96-9.53]). The Kaplan-Meier curves for ischemic stroke in relation to PAD (ABI $\leq 0.99)$, PCA, and normal ABI are also illustrated (Figure 2).

Table 1: Patient demographics and clinical characteristics by ABI group.

\begin{tabular}{|c|c|c|c|c|c|}
\hline & Low ABI $(n=17,091)$ & Normal $(n=17,672)$ & Elevated ABI $(n=5,071)$ & Total $(n=39,834)$ & p-Value \\
\hline Age & & & & & $<0.0001^{a}$ \\
\hline Mean, SD & $69.1(12.7)$ & $62.2(15.1)$ & $71.0(12.4)$ & $66.3(14.7)$ & \\
\hline Range & $18.0,112.0$ & $18.0,102.0$ & $22.0,103.0$ & $18.0,112.0$ & \\
\hline Sex, n (\%) & & & & & $<0.0001^{b}$ \\
\hline Female & $7,286(42.6 \%)$ & $8,199(46.4 \%)$ & $1,480(29.2 \%)$ & $16,965(42.6 \%)$ & \\
\hline Male & $9,805(57.4 \%)$ & $9,473(53.6 \%)$ & $3,591(70.8 \%)$ & $22,869(57.4 \%)$ & \\
\hline Self-reported race, $n(\%)$ & & & & & $<0.0001^{b}$ \\
\hline White & $15,101(88.4 \%)$ & $15,769(89.2 \%)$ & $4,389(86.6 \%)$ & $35,259(88.5 \%)$ & \\
\hline American Indian/Alaskan Native & $60(0.4 \%)$ & $80(0.5 \%)$ & $40(0.8 \%)$ & $180(0.5 \%)$ & \\
\hline Asian & $55(0.3 \%)$ & $132(0.7 \%)$ & $18(0.4 \%)$ & $205(0.5 \%)$ & \\
\hline Black/African American & $216(1.3 \%)$ & $173(1.0 \%)$ & $57(1.1 \%)$ & $446(1.1 \%)$ & \\
\hline Native Hawaiian/Pacific Island & $5(0.0 \%)$ & $8(0.0 \%)$ & $4(0.1 \%)$ & $17(0.0 \%)$ & \\
\hline Other & $186(1.1 \%)$ & $272(1.5 \%)$ & $103(2.0 \%)$ & $561(1.4 \%)$ & \\
\hline Unknown & $1,468(8.6 \%)$ & $1,238(7.0 \%)$ & 460 (9.1\%) & $3,166(7.9 \%)$ & \\
\hline \multicolumn{6}{|l|}{ Comorbidities, n (\%) } \\
\hline Hypertension & $13,618(79.7 \%)$ & $11,287(63.9 \%)$ & $4,236(83.5 \%)$ & $29,141(73.2 \%)$ & $<0.0001^{b}$ \\
\hline Hyperlipidemia & $11,384(66.6 \%)$ & $10,124(57.3 \%)$ & $3,266(64.4 \%)$ & $24,774(62.2 \%)$ & $<0.0001^{b}$ \\
\hline Myocardial infarction & $2,918(17.1 \%)$ & $1,557(8.8 \%)$ & 994 (19.6\%) & $5,469(13.7 \%)$ & $<0.0001^{b}$ \\
\hline Diabetes mellitus & $6,655(38.9 \%)$ & $5,247(29.7 \%)$ & $3,485(68.7 \%)$ & $15,387(38.6 \%)$ & $<0.0001^{b}$ \\
\hline Past or present smoker & $8,991(52.6 \%)$ & $7,100(40.2 \%)$ & 1767 (34.8\%) & $17,858(44.8 \%)$ & $<0.0001^{b}$ \\
\hline TIA & $4,154(24.3 \%)$ & $2048(11.6 \%)$ & 897 (17.7\%) & $5,673(14.2 \%)$ & $<0.0001^{b}$ \\
\hline CKD (stage $\geq 3$ ) & $4,154(24.3 \%)$ & $2,712(15.3 \%)$ & $2,144(42.3 \%)$ & $9,010(22.6 \%)$ & $<0.0001^{b}$ \\
\hline Prior ischemic stroke & $766(4.5 \%)$ & 537 (3.0\%) & $332(6.5 \%)$ & $1,635(4.1 \%)$ & $<0.0001^{b}$ \\
\hline Atrial fibrillation & $4,522(26.5 \%)$ & $3,441(19.5 \%)$ & 1990 (39.2\%) & $9,953(25.0 \%)$ & $<0.0001^{b}$ \\
\hline Coronary artery disease & $10,898(63.8 \%)$ & $7,312(41.4 \%)$ & $3,478(68.6 \%)$ & $21,688(54.4 \%)$ & $<0.0001^{b}$ \\
\hline Carotid artery disease & $5,216(30.5 \%)$ & $2,120(12.0 \%)$ & $1,038(20.5 \%)$ & $8,374(21.0 \%)$ & $<0.0001^{b}$ \\
\hline
\end{tabular}

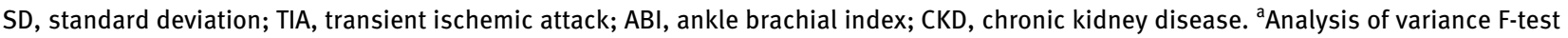
p-value; ${ }^{b} \mathrm{Chi}$-Square $\mathrm{p}$-value. 


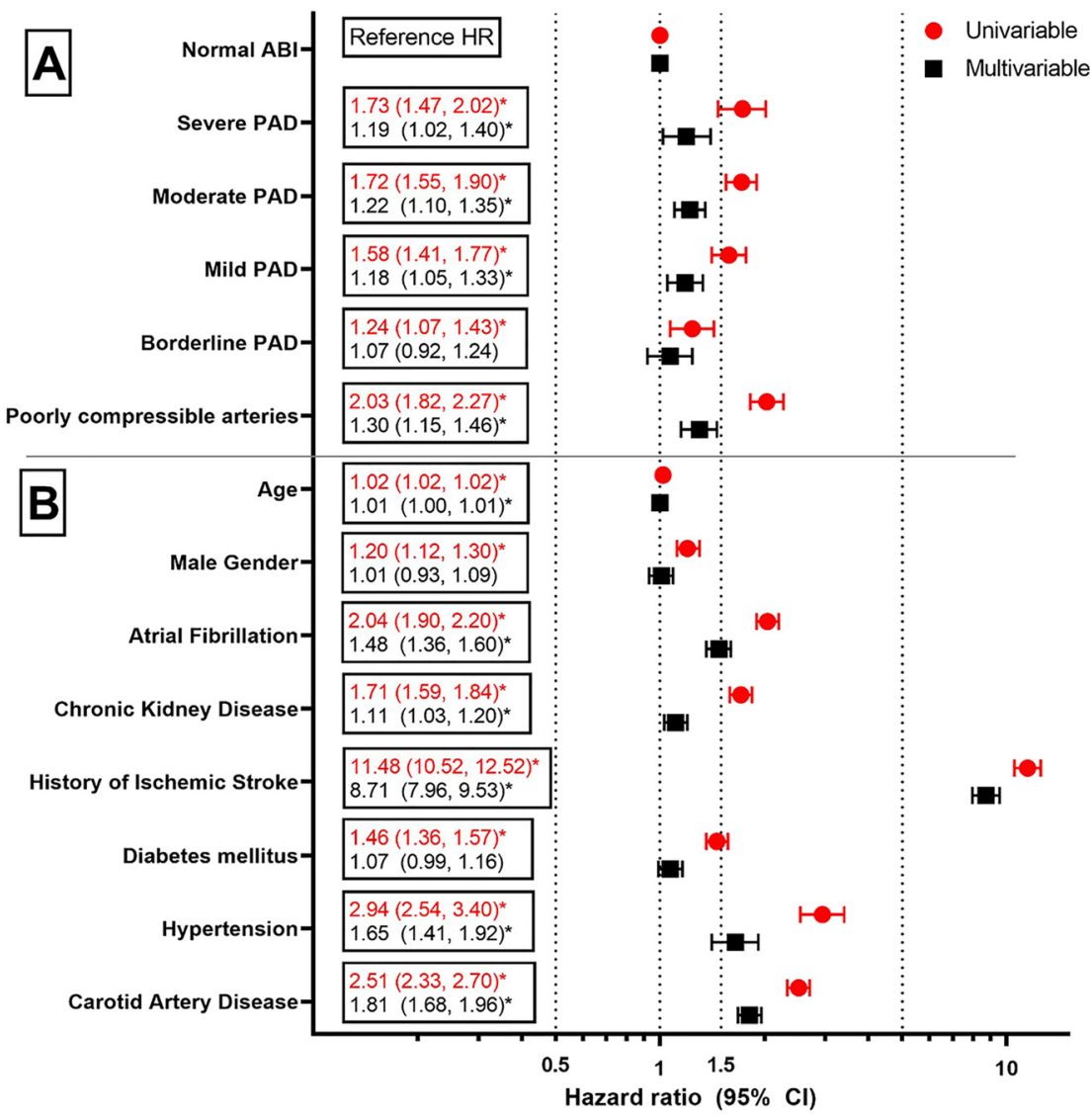

Figure 1: Hazard ratios of incident ischemic stroke by (A) $A B I$ group and (B) risk factor. Multivariable model adjusted for age, gender, atrial fibrillation, chronic kidney disease, prior ischemic stroke, diabetes mellitus, hypertension, and carotid artery disease. ${ }^{*} p<0.05$.

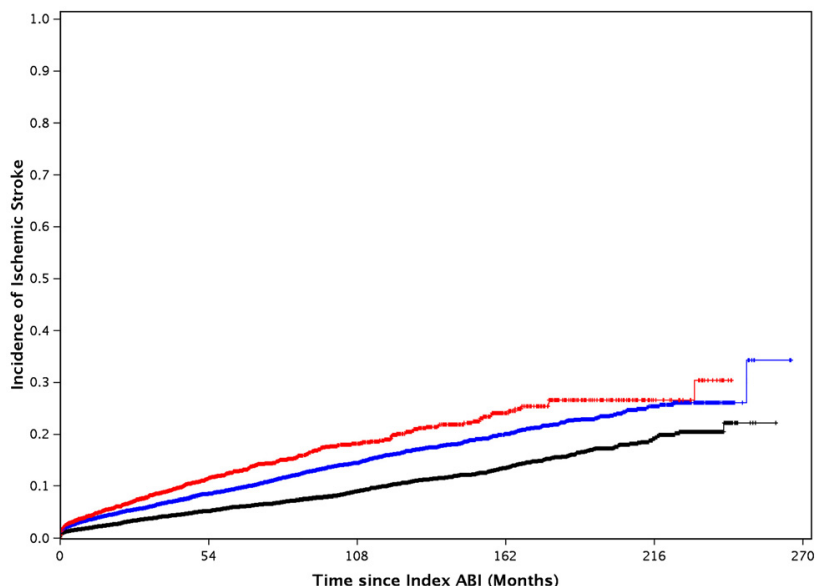

Figure 2: Reverse Kaplan-Meier curves for incident ischemic stroke by low and elevated $A B I$ compared with normal $A B I$. Black, normal $A B I$; blue, $P A D$ or low $A B I$; red, $P C A$ or elevated $A B I$.

\section{All cause mortality by $A B I$ group}

Over one-third $(35.8 \% ; 14,249)$ of the cohort died during the study period. Univariate analysis showed that all abnormal ABI groups were significantly associated with all cause mortality. The greatest all cause mortality risk compared with normal ABI was seen in severe PAD (HR, 3.61 [95\% CI, 3.39-3.84]) and PCA (HR, 3.58 [95\% CI, 3.41-3.76]). History of prior ischemic stroke conferred the greatest risk (HR, 1.81 [95\% CI, 1.68-1.94]) for mortality of the adjusted variables.

On multivariable analysis, the hazard ratios remained statistically significant for all abnormal ABI groups. All cause mortality was most strongly associated with severe PAD (HR, 3.07 [95\% CI, 2.88-3.27]) and PCA (HR, 2.71 [95\% CI, 2.57-2.85]). The ABI groups conferred mortality risk in a PAD severity-dependent manner. All hazard ratios are summarized in Figure 3.

\section{Discussion}

We investigated the predictive value of different $A B I$ groups for ischemic stroke and all cause mortality in a large patient cohort. Previous studies have shown an increased association between low ABI and stroke, but with some 


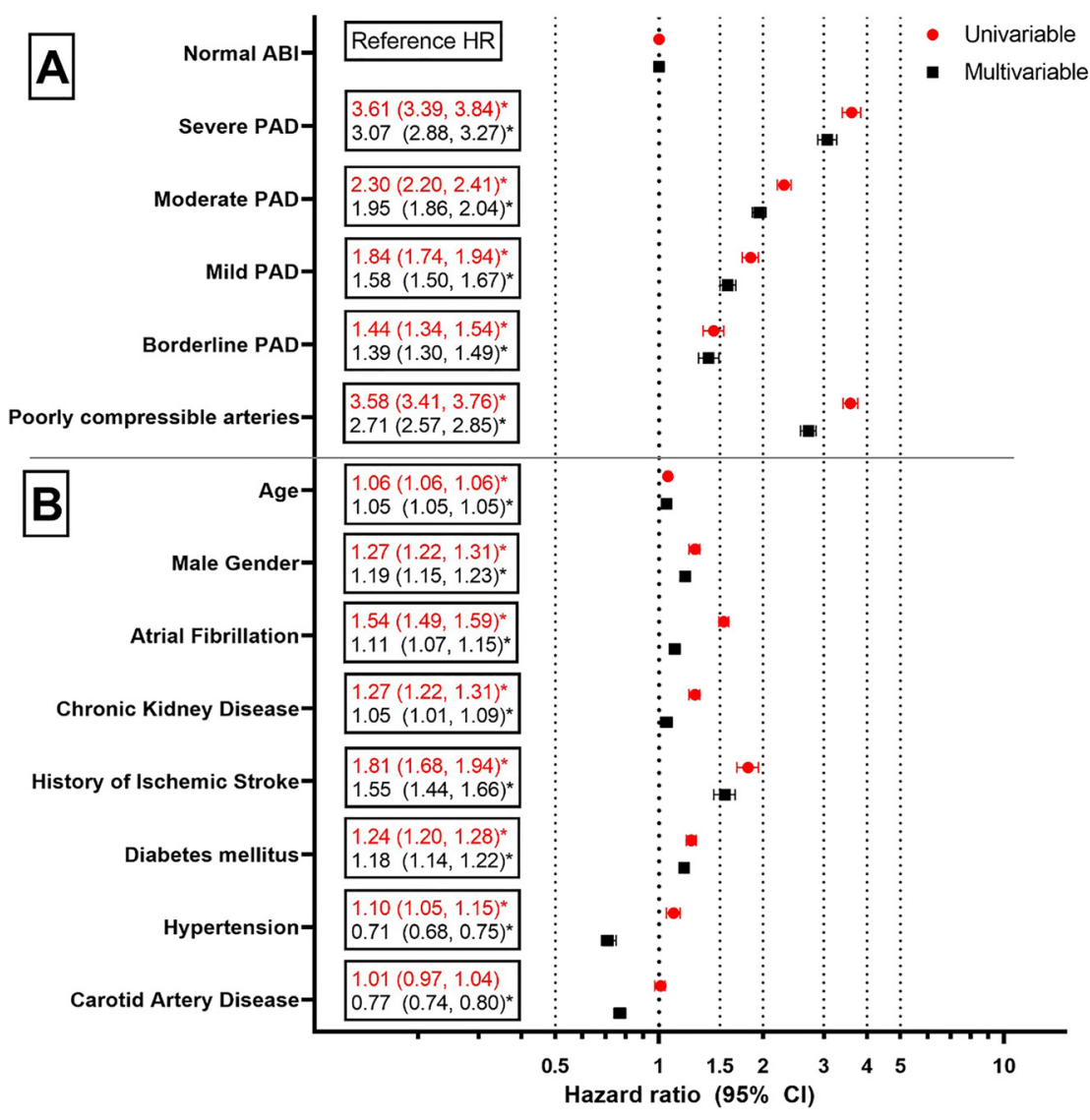

Figure 3: Hazard ratios for all cause mortality by (A) $A B I$ group and (B) risk factor. Multivariable model adjusted for age, gender, atrial fibrillation, chronic kidney disease, prior ischemic stroke, diabetes mellitus, hypertension, and carotid artery disease. ${ }^{*} \mathrm{p}<0.05$. limitations. The Multi-Ethnic Study of Atherosclerosis (MESA) study [18] showed an increased association of $\mathrm{ABI}<1.0$ with composite endpoints including coronary heart disease, stroke, and other vascular events, but not with stroke alone. The 2003 Framingham study [24] revealed the association between PAD and stroke or TIA in an elderly cohort (HR, 2.0 [95\% CI, 1.1-3.7]), but the study was limited by small cohort size (674). The Atherosclerosis Risk in Communities (ARIC) study [25] with a large cohort size of 14,839 participants showed that $\mathrm{ABI}<0.80$ was strongly associated with increased 7-year risk of ischemic stroke (HR, 5.68 [95\% CI, 2.77-11.66]); however, the risk became statistically insignificant (HR, 1.93 [95\% CI, 0.784.78]) after adjusting for major cardiovascular risk factors. This may be because of relatively low number of incident ischemic stroke events (206) compared with our study (453).

A 2014 study by Gronewold et al. [11] showed that low $\mathrm{ABI}(<0.9)$ increased stroke risk in a large population-based cohort $(n=4,299)$, but the impact of PAD severity by ABI was not assessed. Another post-hoc analysis study [7] of the Effects of Ticagrelor and Clopidegrol in Patients with
Peripheral Artery Disease (EUCLID) trial published in 2019 reported an increased ischemic stroke risk in symptomatic PAD patients (HR, 1.31 [95\% CI, 1.04-1.65]). That study [7] included patients with symptomatic PAD with $\mathrm{ABI}<0.60$, whereas our study included both symptomatic and asymptomatic patients of multiple ABI categories who were clinically indicated to undergo ABI measurement. While it is difficult to directly compare the two studies because of different inclusion criterion, the risks of ischemic stroke remain largely consistent. The reason for similar ischemic stroke risk in severe and moderate PAD group in our study could possibly be due to more active management of severe PAD patients. Our study adds to the existing literature as we assess the risk of ischemic stroke alone in a large patient cohort with varying degrees of PAD severity including PCA.

Previous studies of cerebrovascular and mortality risks associated with PCA have been less definitive than those with PAD [10, 18, 26]. The MESA study [18] showed a trend toward increased risk of stroke associated with PCA, but the risk was not statistically significant. In another study [26] of 7,538 high-risk patients from the Second 
Manifestations of Arterial Disease (SMART) cohort, PCA was associated with increased risk of myocardial infarction (HR, 1.83 [95\% CI, 1.22-2.76]) but not with increased risk of stroke (HR, 0.86 [95\% CI, 0.44-1.69]), all cause mortality, or cardiovascular mortality. Similarly, the Cardiovascular Health Study [10] demonstrated increased risk of all cause mortality but not with adverse cardiovascular events, which included stroke in elderly patients with PCA.

In contrast, we report PCA as an independent risk factor for both ischemic stroke and all cause mortality. These discrepant findings may be because some of the previous studies either had smaller populations of patients with PCA $[10,18]$ or had relatively younger patients [11, 26], which may have contributed to lower rates of ischemic stroke. A nationwide cross-sectional study [13] did find increased odds of stroke (OR 1.66 [95\% CI, 1.07-2.56]) in the elevated ABI ( $\geq 1.4$ ) group, but the study was limited by a relatively small sample size (296). Comparatively, we investigated a larger $(5,071)$ and older (mean age, 71.0 [standard deviation (SD), \pm 12.4 years]) PCA cohort with a relatively high incidence of ischemic stroke outcomes (453). Another study [14] with a more comparable cohort (2,781 patients; mean [SD] age, $71.3 \pm 12.1$ years) also demonstrated increased risk of mortality (HR, 2.0 [95\% CI, 1.8-2.2]) in the PCA group compared with the normal ABI group. Patients with PCA have been previously reported to have greater prevalence of diabetes [13] and chronic kidney disease [14]. Although we adjusted for these comorbidities, a residual confounding effect is possible which may explain the increased ischemic stroke risk.

Our results align with previous studies $[9,12,17]$ that showed a severity-dependent relationship with PAD for all cause mortality. Severe PAD and PCA were associated with the highest risk for total death. One possible explanation is that while PAD and PCA are consequences of different pathophysiologic mechanisms, with $\mathrm{PAD}$ resulting from inflammatory processes and atherosclerotic plaque formation [27] and PCA resulting from medial arterial calcification commonly seen among diabetics [19], both are indicators of underlying comorbidities predisposing patients to increased risk of death. Another possible explanation could be that changes in arterial compliance in PAD and PCA patients could indicate abnormal viscerosomatic reflexes [28], which could lead to adverse cerebrovascular outcomes. Therefore, it is crucial for both osteopathic and allopathic physicians providing holistic and integrative cardiovascular and neurological preventative care to screen patients for PAD, which is simple in the clinic setting via an $A B I$ measurement. Health care providers should focus on evaluating a patient's physical, mental, and social wellbeing to promote a healthy lifestyle. Our body's physiologic ability to self-heal can be leveraged by implementing lifestyle changes to help reverse underlying atherosclerotic processes that lead to vascular complications [29, 30]. A targeted screening of high-risk individuals with abnormal ABI can help in pursuing closer monitoring, if necessary, to minimize ischemic stroke risk and all cause mortality.

In addition to continued focus on nonpharmacological interventions such as exercise, smoking cessation, and healthy diet for improved morbidity and mortality outcomes, there has been a growing interest in the role of pharmaceutical prophylaxis to prevent adverse cardiovascular events in PAD patients. Current guidelines recommend using antiplatelet agents, such as aspirin and P2Y12 inhibitors, to reduce major cardiovascular and limb outcomes [8]. Previous studies have evaluated the use of anticoagulants in PAD [31, 32]. The 2017 COMPASS trial [31] showed that rivaroxaban $2.5 \mathrm{mg}$ twice daily with aspirin $100 \mathrm{mg}$ once daily decreased composite events of cardiovascular death, stroke, or myocardial infarction compared with aspirin alone (HR, 0.76 [95\% CI, 0.66-0.86]), but this benefit came at the cost of an increased risk of major bleeding events (HR, 1.70 [95\% CI, 1.40-2.05]). Based on the COMPASS trial findings, the 2020 VOYAGER-PAD [32] study showed that in PAD patients who underwent revascularization, the rivaroxaban and aspirin group reduced composite events of acute limb ischemia, major amputation due to vascular causes, MI, ischemic stroke, or cardiovascular death compared with aspirin alone (HR, 0.85 [95\% CI, 0.76-0.96]).

The use of anticoagulants in PAD patients as standard of care is still under debate in general, but use may be more justified in higher-risk groups. Our study demonstrated that risk of adverse outcomes increased with PAD severity and PCA, which should be further explored, including in regard to more intensive early therapeutic intervention.

\section{Limitations}

This study had several limitations. The data collected in a retrospective study is dependent on a provider recording the given variables. This allows for potentially inconsistent data measurement and misattribution of diagnoses between providers in addition to variable patient follow-up. The study variables and outcomes were extracted using ICD codes from the EHR, which also 
underwent reclassification between the 9th and 10th editions. Furthermore, the cohort was derived from a single healthcare institution and was predominantly comprised of White patients, which limits the generalizability of our findings. Finally, our study only assessed outcomes based on resting $\mathrm{ABI}$ values and rather than PAD symptoms.

\section{Conclusions}

We demonstrated in a large patient cohort that both PAD and PCA were independent risk factors for ischemic stroke and all cause mortality. The risk of ischemic stroke increased in a severity-dependent manner with moderate and severe PAD conferring similar risks. $\mathrm{ABI}$ is a simple and reliable diagnostic tool that can be used to risk stratify patients and may guide further care management based on PAD severity. Further studies are needed to identify patients with abnormal ABI that may benefit the most from aggressive pharmacological intervention in addition to following a healthy lifestyle.

Research funding: None reported.

Author contributions: All authors provided substantial contributions to conception and design, acquisition of data, or analysis and interpretation of data; all authors drafted the article or revised it critically for important intellectual content; all authors gave final approval of the version of the article to be published; and all authors agree to be accountable for all aspects of the work in ensuring that questions related to the accuracy or integrity of any part of the work are appropriately investigated and resolved.

Competing interests: None reported.

Informed consent: The informed consent requirement was waived due to the retrospective, noninvasive nature of the study.

Ethical approval: The study complied with the standards of and was approved by the Mayo Clinic Institutional Review Board.

\section{References}

1. Sen S, Lynch DR, Kaltsas E, Simmons J, Tan WA, Kim J, et al. Association of asymptomatic peripheral arterial disease with vascular events in patients with stroke or transient ischemic attack. Stroke 2009;40:3472-7.

2. Moussa ID, Jaff MR, Mehran R, Gray W, Dangas G, Lazic Z, et al. Prevalence and prediction of previously unrecognized peripheral arterial disease in patients with coronary artery disease: the peripheral arterial disease in interventional patients study. Cathet Cardiovasc Interv 2009;73:719-24.

3. Hong JB, Leonards CO, Endres M, Siegerink B, Liman TG. AnkleBrachial Index and recurrent stroke risk. Stroke 2016;47:317-22.

4. Banerjee A, Fowkes FG, Rothwell PM. Associations between peripheral artery disease and ischemic stroke: implications for primary and secondary prevention. Stroke 2010;41:2102-7.

5. Tziomalos K, Giampatzis V, Bouziana S, Pavlidis A, Spanou M, Papadopoulou $M$, et al. Predictive value of the ankle brachial index in patients with acute ischemic stroke. Vasa 2014;43: 55-61.

6. Topakian R, Nanz S, Rohrbacher B, Koppensteiner R, Aichner FT. High prevalence of peripheral arterial disease in patients with acute ischaemic stroke. Cerebrovasc Dis 2010;29:248-54.

7. Kolls BJ, Sapp S, Rockhold FW, Jordon JD, Dombrowski KE, Fowkes $\mathrm{FGR}$, et al. Stroke in patients with peripheral artery disease. Stroke 2019;50:1356-63.

8. Gerhard-Herman MD, Gornik HL, Barrett C, Barshes NR, Corriere MA, Drachman DE, et al. 2016 AHA/ACC guideline on the management of patients with lower extremity peripheral artery disease: executive summary: a report of the American College of Cardiology/American Heart Association task force on clinical practice guidelines. Circulation 2017;135:e686-e725.

9. Kim ESH, Wattanakit K, Gornik HL. Using the ankle-brachial index to diagnose peripheral artery disease and assess cardiovascular risk. Cleve Clin J Med 2012;79:651-61.

10. O’Hare AM, Katz R, Shlipak MG, Cushman M, Newman AB. Mortality and cardiovascular risk across the ankle-arm index spectrum. Circulation 2006;113:388-93.

11. Gronewold J, Hermann DM, Lehmann N, Kroger K, Lauterbach K, Berger K, et al. Ankle-brachial index predicts stroke in the general population in addition to classical risk factors. Atherosclerosis 2014;233:545-50.

12. Resnick HE, Lindsay RS, McDermott MM, Devereux RB, Jones KL, Fabsitz RR, et al. Relationship of high and low ankle brachial index to all-cause and cardiovascular disease mortality. Circulation 2004;109:733-9.

13. Allison MA, Hiatt WR, Hirsch AT, Coll JR, Criqui MH. A high anklebrachial index is associated with increased cardiovascular disease morbidity and lower quality of life. J Am Coll Cardiol 2008;51:1292-8.

14. Arain FA, Ye Z, Bailey KR, Chen Q, Liu G, Leibson CL, et al. Survival in patients with poorly compressible leg arteries. J Am Coll Cardiol 2012;59:400-7.

15. Lilly SM, Jacobs DR Jr., Kronmal R, Bluemke DA, Criqui M, Lima J, et al. Arterial compliance across the spectrum of ankle-brachial index: the Multiethnic Study of Atherosclerosis. Atherosclerosis 2014;233:691-6.

16. Wilkins JT, McDermott MM, Liu K, Chan C, Criqui MH, Lloyd-Jones DM. Associations of noninvasive measures of arterial compliance and ankle-brachial index: the Multi-Ethnic Study of Atherosclerosis (MESA). Am J Hypertens 2012;25:535-41.

17. Fowkes FG, Murray GD, Butcher I, Heald CL, Lee RJ, Chambless LE, et al. Ankle brachial index combined with Framingham Risk Score to predict cardiovascular events and mortality: a meta-analysis. JAMA 2008;300:197-208.

18. Criqui MH, McClelland RL, McDermott MM, Allison MA, Blumenthal RS, Aboyans V, et al. The ankle-brachial index and incident cardiovascular events in the MESA (Multi-Ethnic Study of Atherosclerosis). J Am Coll Cardiol 2010;56:1506-12. 
19. Ratchford EV, Salameh MJ. Diagnostic approach to peripheral artery disease. In: Slouvut DP, Dean SM, Jaff MR, Schneide PA, editors. Comprehensive review in vascular and endovascular medicine. Minneapolis, MN: Cardiotext Publishing; 2012.

20. Intersocietal Accreditation Commission. IAC standards and guidelines for vascular testing accreditation. Available from: https://www.intersocietal.org/vascular/standards/ IACVascularTestingStandards2020.pdf [Accessed 21 Feb 2021].

21. World Health Organization (WHO). International classification of diseases: ninth revision, basic tabulation list with alphabetic index. Available from: https://apps.who.int/iris/handle/10665/ 39473 [Accessed 21 Feb 2021].

22. World Health Organization (WHO). ICD-10: international statistical classification of diseases and related health problems: tenth revision. Available from: https://apps.who.int/iris/ handle/10665/42980 [Accessed 21 Feb 2021].

23. Benjamin EJ, Muntner P, Alonso A, Bittencourt MS, Callaway CW, Carson AP, et al. Heart disease and stroke statistics-2019 update: a report from the American Heart Association. Circulation 2019;139:e56-e528.

24. Murabito JM, Evans JC, Larson MG, Nieto K, Levy D, Wilson PWF. The ankle-brachial index in the elderly and risk of stroke, coronary disease, and death: the Framingham Study. Arch Intern Med 2003;163:1939-42.

25. Tsai AW, Folsom AR, Rosamond WD, Jones DW. Ankle-brachial index and 7-year ischemic stroke incidence: the ARIC study. Stroke 2001;32:1721-4.
26. Hendriks EJE, Westerink J, de Jong PA, de Borst GJ, Nathoe HM, Mali WP, et al. Association of high ankle brachial index with incident cardiovascular disease and mortality in a high-risk population. Arterioscler Thromb Vasc Biol 2016;36:412-17.

27. Olin JW, Sealove BA. Peripheral artery disease: current insight into the disease and its diagnosis and management. Mayo Clin Proc 2010;85:678-92.

28. Bath M, Owens J. Physiology, viscerosomatic reflexes. In: StatPearls. Treasure Island (FL): StatPearls Publishing; 2020.

29. Parsons C, Agasthi P, Mookadam F, Arsanjani R. Reversal of coronary atherosclerosis: role of life style and medical management. Trends Cardiovasc Med 2018;28:524-31.

30. Johnson B. Tenets of osteopathic medicine. Available from: https://osteopathic.org/about/leadership/aoa-governancedocuments/tenets-of-osteopathic-medicine [Accessed $15 \mathrm{Dec}$ 2020].

31. Eikelboom JW, Connolly SJ, Bosch J, Dagenais GR, Hart RG, Shestakovska 0, et al. Rivaroxaban with or without aspirin in stable cardiovascular disease. N Engl J Med 2017;377: 1319-30.

32. Bonaca MP, Bauersachs RM, Anand SS, Debus ES, Nehler MR, Patel MR, et al. Rivaroxaban in peripheral artery disease after revascularization. N Engl J Med 2020;382:1994-2004.

Supplementary Material: The online version of this article offers supplementary material (https://doi.org/10.1515/jom-2020-0290). 